\title{
Naturaleza y concepto del Derecho a los ojos de Rodríguez-Blanco. Un análisis naturalista de su propuesta metodológica
}

\author{
The Nature and Concept of Law in the Eyes of \\ Rodríguez-Blanco. A Naturalistic Analysis of Her \\ Methodological Proposal
}

Alejandro Guevara Arroyo*

Recepción: 11/03/2020

Evaluación: 21/04/2020

Aceptación final: 02/05/2020

Resumen: En el trabajo Rastreando las críticas de Finnis al punto de vista interno de Hart: inestabilidad y el "sentido y propósito" de la acción humana en el derecho, la iusfilósofa Verónica Rodríguez-Blanco continúa el desarrollo de su concepción sobre la naturaleza y el concepto del derecho, profundizando la metodología necesaria para darle solución a esos asuntos. La mayor parte del texto que presento a continuación se dedica a reconstruir las ideas de Rodríguez-Blanco sobre estos asuntos fundamentales y articular sus ideas con la metateoría de los conceptos científicos y varios de sus problemas metodológicos centrales. Todo esto me permite además enunciar algunas críticas puntuales en contra de la posibilidad de que la metodología de Rodríguez-Blanco para identificar el concepto de Derecho brinde razones epistemológicamente relevantes para sustentar que el concepto así identificado presenta la naturaleza del Derecho.

Palabras clave: naturaleza del Derecho, concepto del Derecho, metodología, conceptos clasificatorios, Finnis.

* Doctorando en Derecho, área Filosofía del Derecho de la Universidad de Buenos Aires, Argentina. Correo electrónico: alguear7@gmail.com 
Alejandro Guevara Arroyo

Abstract: On Tracing Finnis's criticism of Hart's Internal Point of View: Instability and the 'Point' of Human Action in Law, the philosopher Verónica Rodríguez-Blanco continues the development of her conception of the nature and concept of law, deepening the necessary methodology to solve these issues. Most of the text I present below is dedicated to reconstructing Rodríguez-Blanco's ideas on these fundamental issues and articulating her ideas with the metatheory of scientific concepts and several of its central methodological problems. All this also allows me to state some critics against the possibility that the methodology of Rodríguez-Blanco to identify the concept of Law provides epistemologically relevant reasons to support that the concept thus identified presents the nature of the Law. Keywords: nature of Law, concept of Law, methodology, classificatory concepts, Finnis.

\section{Introducción}

Un asunto fundamental de la teoría del derecho es la investigación sobre su naturaleza. En las últimas décadas (y en especial, en la iusfilosofía anglosajona) se ha entendido que tal investigación está emparentada con la de establecer o identificar el concepto del Derecho. Hay muchas versiones de en qué consisten tales cuestiones y cuál es su relación. En esta intervención busco analizar críticamente la solución de Verónica Rodríguez-Blanco ${ }^{1}$ a estos asuntos, tal y como los presenta en el artículo Rastreando las críticas de Finnis al punto de vista interno de Hart: inestabilidad y el "sentido y propósito" de la acción humana en el derecho y obras previas ( $v . g$. Rodríguez-Blanco, 2007, pp. 257-283). Para decirlo en forma lacónica: me interesa cuestionar que la metodología de Rodríguez-Blanco para identificar el concepto de Derecho brinde bases epistemológicamente relevantes para considerar que el concepto así constituido muestra la naturaleza del

1 En su línea de trabajo Rodríguez-Blanco aplica la filosofía de la acción a los problemas de teoría del derecho y otros afines, abrevando de múltiples fuentes teóricas dentro de las que cabe mencionar a Tomás de Aquino, Aristóteles, Wittgenstein, Anscombe y el ius-naturalista neotomista John Finnis. 
Naturaleza y concepto del Derecho a los ojos de Rodríguez-Blanco. Un análisis...

derecho o lo que el derecho es. Sin embargo, para decir algo sobre esto, estimo que podría ser útil presentar algunas cuestiones previas sobre la metateoría de los conceptos - en general- y de los conceptos científicos -en especial-. ${ }^{2}$

El esquema de la ponencia es el siguiente: en primer lugar, presentaré la solución de la iusfilósofa al problema de cuál es el objetivo epistémico de la teoría general del derecho. En segundo término, su respuesta al asunto de cuáles son los pasos o el método necesario y suficiente para satisfacer adecuadamente el mentado fin. Luego señalaré algunos pocos elementos de la metateoría de los conceptos clasificatorios y los aplicaré a los desarrollos de Rodríguez-Blanco. Finalmente, a la luz de esta reconstrucción, puntearé varias críticas que me parece afectan el estatus epistémico de la propuesta.

\section{El objetivo epistémico de la teoría del derecho a los ojos de Rodríguez-Blanco}

En las siguientes líneas intentaré sintetizar el núcleo de la propuesta de Rodríguez-Blanco.

1. La teoría general del derecho busca presentar la naturaleza del Derecho o lo que el derecho es. ${ }^{3}$ En otras palabras, la teoría general del derecho pretende identificar las propiedades de un cierto sector del universo mundo, específicamente acotado al mundo de lo social-humano.

2 Sospecho que algunas de las críticas y consideraciones metateóricas que presentaré podrían extenderse a otras concepciones de la iusfilosofía anglosajona sobre el concepto del derecho. Leiter ha realizado un trabajo semejante en su (Leiter, 2012). En algún sentido, lo que se presenta en esta investigación supone, al menos en alguna medida, una dirección que podría caer bajo el impreciso paraguas que en el mundo anglosajón ha dado en llamarse naturalismo. Para una visión panorámica de las poco precisas líneas que caracterizan las concepciones naturalistas, puede verse (Papineau, 2020); el cuadro general de distintas corrientes y perspectivas naturalistas en la filosofía del derecho puede verse en (Leiter y Etchemendy, 2017).

3 "[L]a pregunta principal acerca de la jurisprudencia sustantiva, a saber, qué es el derecho" (Rodríguez-Blanco, 2021, sección 1). 
1.1. Identificar la naturaleza del derecho es equivalente a dar una respuesta satisfactoria a la pregunta de qué es el derecho (Rodríguez-Blanco, 2021, sección 1 y Rodríguez-Blanco, 2007, pp. 257 y 259$).{ }^{4}$

1.2. Responder qué es el Derecho, a su vez, es equivalente a formular un concepto de Derecho (Rodríguez-Blanco, 2021, sección 1).

2. Ahora bien, es obvio que no cualquier respuesta al problema de la naturaleza del Derecho es una respuesta correcta. La metodología de la teoría del derecho trata sobre los procedimientos y criterios por medio de los cuales se logra que una cierta propuesta de concepto de Derecho pueda ser considerada correcta o adecuada. ${ }^{5}$ El problema metodológico es, obviamente, cuáles son tales pasos o procedimientos. Veamos entonces cuál es el método propuesto por Rodríguez-Blanco para solucionar este asunto.

\section{La concepción metodológica de Rodríguez-Blanco}

Siguiendo en este punto a John Finnis, Rodríguez-Blanco asume una metodología práctico-explicativa (“ $[\mathrm{M}]$ ethodology: an explanatory aspect and a practical one”, Rodríguez-Blanco, 2021, sección 1), una metodología que habilita no solo para solucionar el problema del concepto del derecho, sino también de otros conceptos sociales (Finnis, 1992, cap. 1). Según esta metodología, el concepto de Derecho se identifica asumiendo un cierto "punto de vista" (en adelante PV). Este PV ha de ser, evidentemente, el adecuado. El PV ha de identificar el factor clave señalado por el concepto de Derecho,

4 Señala Rodríguez-Blanco: "Finnis defiende la visión de que cualquier descripción y explicación de lo que sea el derecho debería hacerse desde el punto de vista del hombre que posee razonabilidad práctica. En otras palabras, la razonabilidad práctica nos permite entender las cualidades únicas del derecho [...]" (2021, sección 1, he agregado la cursiva).

5 En algunos pasajes de Rodríguez-Blanco (2021) y, en especial, en obras previas, pareciera que Rodríguez-Blanco considera que la teoría general del derecho es ante todo una especie del género análisis conceptual. Esta asunción, a nivel metateórico, no es nada trivial, aunque bien es cierto que si la autora continúa defendiéndola, y hasta qué punto, es un asunto menos claro. 
Naturaleza y concepto del Derecho a los ojos de Rodríguez-Blanco. Un análisis...

que consiste en la función normativa de esa institución (Rodríguez-Blanco, 2021, sección 1). La metodología también ha de permitir explicitar cómo el concepto de Derecho da cuenta del hecho de que entre las personas que actúan en la sociedad existen distintas concepciones sobre qué es el Derecho y sobre qué lo integra (Finnis, 1992, p. 38). El concepto de Derecho ha de distinguir entre las concepciones marginales y centrales del Derecho.

Para ello, esta metodología pide atender a la perspectiva interna ${ }^{6}$ del integrante de esa actividad social, o sea, aquel que es normativamente dirigido por ella. ${ }^{7}$ Posicionarse de esa forma supone identificar las asunciones que sobre la estructura normativa de esa institución tiene quien funciona/ agencia en esa práctica (Rodríguez-Blanco, 2021, sección 1). ${ }^{8}$ Esto incluye necesariamente reconocer las distinciones que cualquier persona que participa de esa práctica/institución supone y utiliza (Rodríguez-Blanco, 2021, sección 1). Se habilita así, la persona teórica, a percibir el concepto de Derecho del practicante.

Este reconocimiento del adecuado concepto de Derecho identifica un cierto caso central o significado focal, el cual puede ser utilizado de muchas formas. Por esto, aunque existe un concepto de Derecho, este aparece de distintas formas en distintas concepciones del Derecho (Rodríguez-Blanco, 2021, sección 1).

La ubicación de todo esto se alcanza asumiendo el PV de la persona que posee razonabilidad práctica (en adelante PPRP) (Rodríguez-Blanco, 2021, sección 1): ${ }^{9}$ una persona que tiene hábitos, valores, intenciones y creencias en una comunidad dada. La PPRP funciona (i. e. actúa) adecuadamente en esa práctica, presumiblemente pues posee (= comprende) cuál es el

6 Que no ha de ser confundido con el punto de vista interno en sentido hartiano.

7 "El agente que ejecuta la acción o el participante que participa en la práctica le otorga su sentido y propósito - o valor - a la acción o práctica." (Rodríguez-Blanco, 2021, sección 1). Poco antes: “[...] '[D]erecho' se refiere a un solo concepto, [...] [cuya] fuente primaria, [...] es el punto de vista del hombre que posee razonabilidad práctica" (Rodríguez-Blanco, 2021, sección 1).

8 En un artículo previo se señala: “[P]ractical point of view enables us to identify and determine the subject matter of jurisprudence" (Rodríguez-Blanco, 2007, p. 258).

9 Previamente, afirmó "[L]a formación de un concepto depende de la aprehensión del punto de vista práctico del hombre” (Rodríguez-Blanco, 2021, sección 1). 
objetivo, valor o significado (meaning) de esa práctica y de la institución en que se desenvuelve (Rodríguez-Blanco, 2021, sección 1).

En el artículo analizado, Rodríguez-Blanco propone que entender el punto de vista de quien razonablemente participa en una práctica es equivalente a identificar el logos, objetivo o valor de la acción que realiza y la práctica en que se realiza tal acción. ${ }^{10}$

Empero, y aquí se opone a Herbert Hart, el logos o finalidad de una acción y de una práctica no ha de equipararse con las creencias de quien se encuentra en esa práctica. ${ }^{11}$ Se trata, más bien, de una clase de intención (con c) que da unidad a las acciones que conforman las prácticas (Rodríguez-Blanco, 2021, sección 2). Tal sería el logos intencional inmanente a la práctica (Rodríguez-Blanco, 2021, sección 2). Este logos individualiza las acciones que se dan ahí y se muestra como el conjunto de características remanentes una vez descritos todos los aspectos físicos presentes en una acción (Rodríguez-Blanco, 2021, sección 2). Esta intención está en los practicantes en la forma de cierta clase de conceptos que están en funcionamiento durante la acción en cuestión (i.e. no la anteceden, sino que son determinantes internos de la acción misma (Rodríguez-Blanco, 2021, sección 2). ${ }^{12}$

La profesora propone que para averiguar este trasfondo de distinciones que sirven a la acción intencional ha de apelarse a lo que llama la metodología del porqué, inspirada en la filosofía de la acción de Elizabeth Anscombe. Esta metodología pregunta al agente por qué o cuál es la razón por la que realizó, realiza o realizará cierta acción (Rodríguez-Blanco, 2021, sección 2). Para controlar si la respuesta es genuina, quien la formula ha de evaluarla apelando a un conjunto de condiciones contextuales, que son los conceptos aprehendidos durante la juventud (Rodríguez-Blanco, 2021, sección 2). Solo es posible entender las razones del agente como un

\footnotetext{
10 "La comprensión completa de las acciones y las prácticas implica una comprensión del sentido y propósito de la acción o práctica" (Rodríguez-Blanco, 2021, sección 1).

11 "El estado mental de la creencia” (Rodríguez-Blanco, 2021, sección 2), luego: “[L]os estados mentales que racionalizan las acciones” (Rodríguez-Blanco, 2021, sección 2).

${ }^{12}$ Como se dice en el texto principal, se trata de una concepción legataria del pensamiento de la filósofa Anscombe, como la ius-pensadora reconoce. Anscombe negó que la acción deba concebirse como un efecto causal de estados mentales y deseos que la anteceden (Rodríguez-Blanco, 2021, sección 2).
} 
Naturaleza y concepto del Derecho a los ojos de Rodríguez-Blanco. Un análisis...

caso de una práctica social y de las características de cómo funcionar bien (good-making) en esa práctica. Esto último es lo que conformaría el logos intencional de la práctica (Rodríguez-Blanco, 2021, sección 2). No dedicaré más espacio a este asunto aquí, aunque regresaré a él más adelante.

A partir del uso de esta metodología, la autora, en un artículo previo, sigue a Finnis al decir que el concepto de Derecho adecuado es el de un conjunto de reglas, aseguradas mediante sanciones y creadas por una autoridad efectiva para toda una comunidad, mediante las cuales se resuelven problemas de coordinación en favor del bien común de esa comunidad (Rodríguez-Blanco, 2007, p. 281). ${ }^{13}$ No diré más de este punto, en tanto la solución específica al problema del concepto de Derecho de Rodríguez-Blanco no es el interés principal de estos comentarios.

\section{Metateoría de los conceptos}

En lo que sigue presentaré sucintamente unos pocos aspectos —más bien bastante conocidos- sobre una cierta clase de conceptos: los clasificatorios. ${ }^{14} \mathrm{Me}$ concentraré en esta clase pues creo que a ella pertenece el concepto de Derecho cuya corrección Rodríguez-Blanco pretende sustentar con su metodología.

Luego presentaré algunas características relevantes de los conceptos clasificatorios científicos y varios de los problemas que se presentan al considerar el asunto de su corrección o adecuación.

Finalmente, intentaré una breve reconstrucción metateórica análoga con los desarrollos hasta aquí presentados de la propuesta de Rodríguez-Blanco sobre el concepto de Derecho. Todas estas distinciones meta-

13 Presumo que tal sería el logos de esa práctica denominada Derecho.

14 También llamados taxonómicos o cualitativos. Asumiendo una clasificación bastante extendida, se puede distinguir entre conceptos clasificatorios (que son los más comunes en la vida diaria y de estructura formal más simple), conceptos comparativos y conceptos métricos. Es obvio que otras subdivisiones pueden ser introducidas. Sobre estos puntos, véase Mosterín (2003, pp. 15-44) y Diez \& Moulines (1999, pp. 101-124). Obviamente, asumir esta clasificación no excluye que los conceptos pueden ser ordenados bajo algún criterio distinto. 
teóricas son vitales para expresar con precisión mis dudas sobre la propuesta de la autora. Esta es quizás la razón principal para presentar estos asuntos. Vamos a ello.

\subsection{Fundamentos}

Partamos de que todo concepto (clasificatorio o de otras clases) puede ser analizado en dos elementos: su intensión (con $s$ ) y su extensión. La intensión de un concepto es su contenido. Ahí se expresan o señalan un conjunto de propiedades y/o relaciones (ver Ferrater Mora, entrada comprensión, y Bunge, 2004, p. 59). Por su parte, la extensión del concepto es aquello que cae bajo el concepto. ${ }^{15}$

Extensionalmente considerado, todo concepto clasificatorio establece un conjunto. ${ }^{16}$ Veamos esto con mayor detenimiento: un concepto clasificatorio induce una partición de un dominio dado, estableciendo una relación de equivalencia ${ }^{17}$ pertinente a todos los integrantes del conjunto partición inducido por el concepto (Mosterín, 2003, pp. 20-21). Es obvio que hay distintas formas de hacer una partición de un dominio, o sea, de crear un conjunto (Mosterín, 2003, pp. 106-110). Un concepto clasificatorio preciso lo hace mediante la delimitación de un conjunto de propiedades que aparecen en todos los integrantes del conjunto partición inducido por el concepto. Es claro que el conjunto de propiedades en cuestión es señalado en la intensión del concepto y que el conjunto partición inducido por el

15 Se trata de una distinción habitual para la filosofía de la ciencia. Ver, por ejemplo (Mosterín \& Torreti, 2002, entrada concepto).

16 Véase Diez \& Moulines (1999, pp. 96-97) y Ferrater Mora y Leblanc (1992, pp. 119-122). Ahí se encuentran algunas distinciones relevantes en relación con conceptos intuitivos que se confunde frecuentemente con la noción de clase o conjunto.

${ }^{17}$ Una relación de equivalencia es una relación reflexiva, simétrica y transitiva. Esta es una definición muy habitual. Ver, por ejemplo, De Salama (1996, p. 186). Sobre estas propiedades formales de las relaciones, véase Ferrater Mora y Leblanc (1992, pp. 155-157) y De Salama (1996, pp. 182-185). 
Naturaleza y concepto del Derecho a los ojos de Rodríguez-Blanco. Un análisis...

concepto sobre un dominio dado es equivalente a la extensión del concepto (Mosterín, 2003, 17). ${ }^{18}$

Las anteriores son condiciones metateóricas de adecuación formal de los conceptos clasificatorios en general. Paso ahora a los conceptos científicos clasificatorios.

\subsection{Conceptos científicos clasificatorios, corrección y teorías científicas}

Los conceptos clasificatorios científicos ${ }^{19}$ tienen papel ante todo en dos espacios teóricos: (1) como parte de teorías y modelos explicativos y (2) integrando sistemas conceptuales clasificatorios (Mosterín, 2003, p. 17) ${ }^{20}$ Téngase en cuenta, adicionalmente, que aunque una teoría explicativa incluye conceptos no puede ser reducida a conceptos. ${ }^{21}$ Por su parte,

${ }^{18}$ La reconstrucción lógica de conceptos clasificatorios es sencilla, pues los términos que los expresan son predicados monádicos (Diez \& Moulines, 1999, p. 101).

${ }^{19}$ De pasada mencionaré que un concepto clasificatorio científico puede (o no) ser anclado a un término científico mediante una definición técnico estipulativa $(v \cdot g$. la definición de los términos "galaxia", "protón", "primate").

20 Extensionalmente considerada, una adecuada clasificación ha de cumplir varios requisitos formales, que pueden sintetizarse de la siguiente forma: dado un dominio, todo individuo del dominio ha de pertenecer a un y sólo un conjunto que sea extensión de un concepto y no ha de formularse un concepto para el que al menos un individuo del dominio no pueda pertenecer (Mosterín, 2003, p. 19). Es obvio, por ende, que si bien todo concepto clasificatorio induce un conjunto partición de un dominio, una clasificación propiamente se genera mediante la creación de varios conceptos aplicables al dominio en cuestión (Diez \& Moulines, 1999, p. 101).

${ }^{21}$ Una genuina teoría explicativa incluye más contenido, pues al menos una parte de la teoría pretende ser un modelo de la forma en que un fragmento del mundo deviene. Para ello, es imprescindible que incluya o suponga enunciados que expresan leyes, patrones, mecanismos o regularidades en las formas del cambio y de la determinación entre hechos. De aquellos se pueden inferir diversas implicaciones que - grosso modo- conforman las explicaciones de hechos particulares ( $v$. g. el último movimiento de marte alrededor del sol durante cierto periodo de tiempo, la desaparición de una especie hace millones de años, la caída de un régimen político tal o cual). En sentido semejante, desarrollando la estructura de las leyes (en sentido científico) y sus clases, así como el concepto de explicación y su conexión con las leyes (véase Bunge, 2004, pp. 265-299 y pp. 459-471). Conceptos semejantes son desarrollados por Elster (2010, pp. 21-82), en donde se reconstruyen además otras partes relevantes de las teorías explicativas en las ciencias sociales, como los mecanismos causales y su conexión 
de entre los sistemas conceptuales clasificatorios, los árboles de taxones son los más finos y precisos, ordenando niveles jerárquicos de conceptos clasificatorios.

Ahora bien, además de condiciones formales de adecuación, los conceptos tienen condiciones materiales de adecuación o de corrección o de validez. Propongo que el mostrar que un concepto clasificatorio es materialmente correcto o adecuado es condición necesaria o - quizás-que es equivalente a sustentar que ese concepto muestra la naturaleza de lo que señala.

La corrección o validez material (no formal) de un concepto científico clasificatorio puede ser analizada en diversos aspectos, de los cuales solo mencionaré dos, pues son de interés para lo que luego comentaré sobre el concepto de Derecho y la concepción metodológica de Rodríguez-Blanco.

(a) El primero es lo que llamaré el problema del realismo de las propiedades.

(b) El segundo es el de la arbitrariedad de la clasificación.

Le llamo problema del realismo de las propiedades al asunto de sustentar que las propiedades señaladas en la intensión del concepto efectivamente existen o están presentes en los individuos que pertenecen al conjunto que es equivalente a la extensión del concepto.

Para presentar el segundo problema, notemos lo siguiente. Como ya señalé antes, un conjunto que es partición de un universo o dominio dado puede establecerse de muchas formas. Por ejemplo, puedo hacer una colección o conjunto en este auditorio, formado por los bolígrafos de los conferencistas y el respaldar de las sillas de la primera fila. Es un conjunto y formalmente es tan bueno como cualquier otro. Por su parte, puedo hacer una clasificación de todas las personas de este auditorio de la siguiente forma: un conjunto estará formado por todas las personas de menos de 34 años, otro por las personas de más de 34. Esta clasificación es formalmente precisa, pero claramente nos parece arbitraria.

con la interpretación de las acciones y los comportamientos. Es obvio que hay muchos debates filosóficos sobre el concepto (o conceptos) de explicación científica: para una detallada reconstrucción de los debates clásicos ver Salmon (1992, pp. 7-41). 
Naturaleza y concepto del Derecho a los ojos de Rodríguez-Blanco. Un análisis...

Ahora, en las ciencias es común suponer que muchos de los conceptos clasificatorios y las clasificaciones de un dominio dado no son arbitrarios: hay razones que sustentan su preferibilidad material o epistémica. Identificar estas justificaciones es dar cuenta del problema de la arbitrariedad.

No puedo aquí analizar con detalle estos problemas y sus soluciones. Tengo que restringirme a decir algunas pocas ideas (sin poder darles mayor sustento). Con respecto al primer asunto, una de las principales formas de sustentar la existencia de ciertas propiedades es por la presencia o aceptación de esas propiedades en hechos explicados por teorías y programas científicos explicativos fértiles y progresivos. Por ejemplo, la existencia del éter en sentido aristotélico se vio privada de justificación tras la refutación del programa astronómico y cosmológico aristotélico y - tras ello- que una entidad esté formada de éter o quintaesencia ya no puede considerarse una propiedad real que sea mencionada como parte de la intensión de ningún concepto adecuado.

En relación con el asunto de la arbitrariedad, notemos cómo se abordó con respecto al árbol de taxones biológicos de especie lineana. ${ }^{22}$ En la historia de la biología se discutió bastante sobre la arbitrariedad o naturalidad de este sistema de conceptos clasificatorios. Dos son las respuestas que deseo rescatar. La primera es la de Mosterín, quien señala que este sistema es más natural $-i$. e. no arbitraria- pues mediante los conceptos que lo integran podemos formular leyes fértiles y sólidamente contrastadas sobre los seres vivos y su devenir. ${ }^{23}$ La segunda depende de la teoría de la evolución biológica: como ya se dijo, los conceptos que pertenecen a cada nivel del árbol de taxones lineano establecen una relación de equivalencia entre todos los individuos de la extensión de cada concepto. En este caso, estas relaciones de equivalencia pueden ser sustentadas como naturales (no arbitrarias) pues reflejan el común antepasado evolutivo de los individuos pertenecientes al conjunto extensión de cada concepto clasificatorio. Al tiempo, los niveles más altos de la jerarquía reflejan antepasados comunes

${ }^{22}$ Que - en su versión más simple - está constituido por siete niveles jerarquizados de conceptos clasificatorios: reino, phylum, clase, orden, familia, género y especie.

23 “ ¿Y por qué es natural la clasificación de los animales en filos o phyla (anélidos, moluscos, etc.)? la respuesta es que podemos enunciar muchas e interesantes leyes generales acerca de los anélidos, por ejemplo [...]” (Mosterín, 2003, p. 23). 
más lejanos evolutivamente Así, por ejemplo, los integrantes de la especie homo sapiens comparten un ancestro común. Este antepasado es más cercano evolutivamente que el antepasado compartido por todos los integrantes del género homo (Bowler, 1992, p. 165). Los conceptos clasificatorios y el sistema taxonómico reflejan esas relaciones reales. Pero estas clases de relaciones reales (y su forma de devenir) son explicables sólo por la teoría de la evolución que - como ya dije- es mucho más que un sistema conceptual clasificatorio.

Obviamente, hay mucho más que decir de todos estos asuntos. Por ahora, les pido que se acepten estos breves apuntes metateóricos y se retengan dos ideas claves:

En primer lugar, los conceptos científicos clasificatorios forman parte de teorías explicativas, por ejemplo, dentro de enunciados legaliformes. ${ }^{24}$ También se articulan en sistemas clasificatorios, pero las teorías propiamente explicativas no pueden ser reducidas ni a conceptos clasificatorios ni a sistemas clasificatorios.

En segundo lugar, para sustentar su corrección, validez o adecuación material, los conceptos necesariamente han de apelar a teorías e hipótesis que no son ni conceptos clasificatorios ni sistemas clasificatorios. Las relaciones metodológicas que se presentan entre las teorías e hipótesis explicativas y los mentados conceptos son variadas, pero aquí lo que quiero dejar sentado es que la corrección material de los conceptos clasificatorios parece ser parasitaria del estatus epistémico de teorías y modelos explicativas que son mucho más que aquellos. Esto es clave.

${ }^{24}$ Así, por ejemplo, el concepto de grave forma parte de la ley de caída de los graves de Galileo Galilei, el de energía es necesario en la segunda ley de la termodinámica de la Física o un cierto concepto de Constitución aparece en los mecanismos causales para el cambio constitucional investigados por la ciencia política. 
Naturaleza y concepto del Derecho a los ojos de Rodríguez-Blanco. Un análisis...

\subsection{Una reconstrucción metateórica de las ideas de Rodríguez-Blanco}

Preguntémonos: ¡cuáles son las características metateóricas formales del concepto de Derecho tal y cómo lo identifica la metodología de Rodríguez-Blanco? ¿Tiene el concepto de Derecho alguna relación metodológica importante análoga a la que los conceptos científicos clasificatorios tienen con las teorías explicativas? Y — de especial interés- ¿cómo (desde la concepción de Rodríguez-Blanco) se justifica la adecuación o corrección material de un cierto concepto de Derecho? ¿Apelando a qué? Estas últimas dos preguntas parecen de importancia para satisfacer el objetivo epistémico que interesa a la autora. Recuérdese que el concepto de Derecho aspira a dar cuenta de lo que el Derecho es y que una condición necesaria de que podamos decir que una propuesta del concepto de Derecho identifica la naturaleza de Derecho, es que aquella pueda resolver adecuadamente tanto el problema del realismo de las propiedades como el problema de la arbitrariedad. Pero entonces, el asunto de la justificación epistémica de la adecuación material de un cierto concepto de Derecho es un problema cuya resolución es teóricamente imprescindible.

Lo primero es que el concepto de Derecho es un concepto clasificatorio: establece una distinción entre aquello que es derecho y aquello que no lo es. En la intensión del concepto se señalan las propiedades cuya presencia en un cierto hecho permite considerarlo como perteneciente al conjunto equivalente a la extensión del concepto. Sin embargo, para Rodríguez-Blanco ${ }^{25}-\mathrm{y}$ también para Finnis - el funcionamiento como instrumento clasificatorio del concepto de Derecho es ligeramente distinto al estándar: las personas que participan en la práctica distinguen como Derecho hechos que no siempre comparten todas las propiedades señaladas en la intensión del concepto.

La situación podría ser representada con sencillez de la siguiente forma: Sean BCDE el conjunto de propiedades señaladas en la intensión del concepto de Derecho. Sucede que en ocasiones se dan casos como $\alpha$ : BCD o $\beta$ : DE o $\gamma$ : BE. Todos estos casos son clasificados como Derecho, aunque poseen sólo algunas de las propiedades (y no las mismas, por cierto) seña-

${ }^{25}$ Rodríguez-Blanco (2021, sección 1) y Rodríguez-Blanco (2007, pp. 259-260). 
ladas en la intensión del concepto de Derecho. El concepto de Derecho, dado que funciona de esta forma, pertenece a una clase especial de conceptos clasificatorios, que Finnis intentó articular con la idea aristotélica de significado focal y relación analógica, aunque en un artículo previo Rodríguez-Blanco conectó más bien con la wittgensteniana semejanza nuclear (core resemblance) (Rodríguez-Blanco, 2007, pp. 278-282).

Ahora bien, como se vio anteriormente, los conceptos científicos clasificatorios tienen un papel clave como integrantes de teorías que son más que conceptos clasificatorios. De forma análoga, me parece que el concepto de Derecho - tal y como es teorizado por Rodríguez-Blanco- integraría o formaría parte de concepciones que responden a problemas de cómo y por qué el Derecho - y lo que lo conforma- es como es y funciona en la forma en que lo hace. Estas concepciones incluirían más que el mero concepto clasificatorio del Derecho. Todas estas concepciones y clasificaciones son asumidas por los agentes y, específicamente, por la PPRP al actuar en ciertas prácticas sociales (Rodríguez-Blanco, 2021, sección 1).

Paso ahora a los problemas de la corrección material del concepto. Creo que una dirección consistente con la constelación de ideas de la profesora que puede solucionar estos asuntos podría apelar a las diversas relaciones del concepto del Derecho con concepciones ínsitas en las personas practicantes y, en especial, por la PPRP. Cuáles exactamente podrían ser las relaciones metodológicamente relevantes para responder estos asuntos, sin embargo, no es algo que aquí pueda profundizar. ${ }^{26}$

Cualquiera sea el caso de esto, me parece claro que - según la teorización de la profesora- el método práctico-explicativo tiene la función de identificar el concepto y la concepción nucleares, que serían los de la PPRP. De alguna forma, la persona teórica tendría que representarse o comprender eso que está en la PPRP. En esa empresa, la metodología del porqué profundizaría en los pasos necesarios para representarse o comprender las concepciones/logos intencional (con c) supuestas en el accionar de la PPRP. ${ }^{27}$

${ }^{26}$ No me queda claro si Rodríguez-Blanco tiene una posición sobre estos asuntos metodológicos. Regreso a estas cuestiones en la sección dedicada a mis dudas y críticas.

27 Si esto es correcto, la metodología del derecho de la profesora Rodríguez-Blanco no brindaría los pasos para construir un concepto, sino para reconocerlo. Contrástese con la concepción y críticas de Nino, 2017, cap. IX, intitulado El enfoque esencialista del concepto de Derecho. 
Naturaleza y concepto del Derecho a los ojos de Rodríguez-Blanco. Un análisis...

Hasta aquí estas sugerencias metateóricas sobre las ideas de Rodríguez-Blanco.

\section{Dudas y críticas}

En último lugar presentaré algunas dudas sobre la propuesta de Rodríguez-Blanco tal y como la he reconstruido. Estas dudas podrían también ser entendidas como críticas a esta concepción metodológica.

La primera duda parte de un cierto aspecto difícil de conciliar en varios pasajes del artículo analizado, en especial comparados con investigaciones anteriores de Rodríguez-Blanco. ${ }^{28}$ Se trata del estatuto de las concepciones que poseen quienes agencian en el Derecho y, en específico, de la PPRP. Una concepción pareciera aquí querer decir al menos dos cosas.

En primer lugar, algo así como teorías del sentido común que tratan sobre un cierto fragmento de la realidad y su funcionamiento. En otras palabras, se trataría de folktheories. ${ }^{29}$ Tales teorías serían un conjunto de contenidos de cuya posesión el agente puede que no sea muy consciente, pero que, al fin y al cabo, es proposicional o traducible en conjuntos de enunciados. Esta me parece la opción más intuitiva de la noción de concepción. Sin embargo, esta vía parece ser incompatible con afirmaciones que se encuentran en investigaciones más recientes de la profesora, incluyendo la presente en debate. ${ }^{30}$

La segunda opción es que al decir que los agentes tienen concepciones de la práctica, se alude al logos intencional de las prácticas y todo lo que ahí se incluye. Este no es un contenido proposicional, sino más bien distinciones que constituyen la acción del agente en la práctica social y la

${ }^{28}$ V.g. en su Rodríguez-Blanco, 2003, p. 99-124.y en Rodríguez-Blanco, 2006, p. 26-54 (criticando a Dworkin y Perry).

${ }^{29}$ Sobre cómo identificar las teorías populares o folktheories: "To the extent that our intuitions coincide, they reveal our shared theory and to the extent that our intuitions coincide with those of the folk, they reveal the folk theory" (Rodríguez-Blanco, 2006, p. 38).

${ }^{30}$ Esta opción acercaría la concepción de Rodríguez-Blanco a la de Frank Jackson y su análisis conceptual no ambicioso (Jackson, 1994, p. 23-42.). 
dirigen. No son folktheories, sino que serían utilizadas ciegamente ${ }^{31}$ por el agente en su accionar.

Dependiendo cómo se responda este asunto, se me ocurren dos señalamientos críticos.

En caso de que se asuma que las mentadas concepciones son folktheories en el sentido de contenidos proposicionales, cabría preguntarse por qué estas habrían de considerarse correctas o aceptables. De otra forma: podríamos aceptar que las personas tienen tales teorías, sin aceptar que son formas correctas de entender la realidad. Después de todo, por definición, se trataría de contenidos que no han sido sometidos a procedimientos metódicos de crítica racional. Este estatus epistémico pre-crítico se transmitiría a los conceptos que las integran y cuya corrección depende de ellas, incluyendo el concepto de Derecho focal que ahí se pueda localizar. ${ }^{32} \mathrm{De}$ forma que, si asumimos que un cierto concepto de Derecho integra una folktheorie, no tenemos bases epistemológicamente sólidas para suponer que ese concepto señala la naturaleza del Derecho.

Según me ha aclarado explícitamente la autora, su posición es la segunda. En este caso, aparece una cuestión sutil. Supongamos que disponemos de un procedimiento epistemológicamente sólido ${ }^{33}$ para sostener que un $\alpha$ es el logos intencional asumido por un agente $\beta$, quien participa en la práctica jurídica. Aceptemos, por mor del argumento, que la metodología práctico-explicativa sirve bien para identificar el significado nuclear apelando al PV de la PPRP. Identificamos, así, que $\alpha_{1}$ es el concepto nuclear de Derecho.

Ahora, representémonos la siguiente situación: se formula una potente teoría explicativa, llamémosle $\varepsilon$, que establece sólidas leyes sociales sobre el funcionamiento del Derecho en buena parte del mundo. Esta utiliza

${ }^{31}$ En la respuesta de Rodríguez-Blanco a esta ponencia, ella precisó que no cree que el seguimiento sea ciego, sino que es plenamente reflexivo.

${ }^{32}$ Un punto semejante es señalado por Leiter (2012, p. 246). Por su parte, la iusfilósofa presenta diversas críticas y retos que considera que debe responder el naturalismo a la Leiter en (Rodríguez-Blanco, 2008). No obstante, tengo la impresión que tales críticas - de ser acertadas - impactan al naturalismo ontológico y no al metodológico. Las consideraciones que aquí se han desarrollado caen en esta última clase.

${ }^{33}$ Verbigracia, la metodología del porqué de Anscombe y Rodríguez-Blanco. 
Naturaleza y concepto del Derecho a los ojos de Rodríguez-Blanco. Un análisis...

un concepto $\gamma$ de Derecho, bastante distinto a $\alpha_{1}$. Como si fuera poco, la existencia de las propiedades señaladas en la intensión de $\gamma$ puede ser explicada a partir de potentes programas científicos $\delta$, conectándolas con propiedades más profundas del mundo ( $v . g$. mecanismos neuropsicológicos presentes en los humanos ${ }^{34}$ ).

¿Estaríamos justificados en decir que, en realidad, los problemas de la adecuación material del concepto de Derecho no son correctamente superados por $\gamma$, a pesar de ser un integrante necesario de la fértil y bien contrastada teoría explicativa $\varepsilon$ y de que las propiedades que señala son sólidamente explicables por $\delta$ ? ¿No tendríamos buenas razones para asumir que $\gamma$ es un concepto de Derecho que no es arbitrario y cuyas propiedades realmente existen? En ese caso, ¿por qué no considerar que $\gamma$ reveló lo que el Derecho es o - al menos - una parte? ¿Por qué no sostener que esa es una respuesta sólidamente fundada a la pregunta de qué es el Derecho? Pareciera que tenemos las mejores razones para creer que este concepto señala qué es el Derecho. ¿Qué ventaja epistémica obtendríamos en insistir, en cambio, que sólo $\alpha_{1}$ constituye la naturaleza del Derecho, a pesar que $\alpha_{1}$ no tiene tan relevantes relaciones con teorías explicativas ni puede - apelando a la metodología de Rodríguez-Blanco - invocar razones tan epistemológicamente potentes como las brindadas mediante los criterios de justificación epistémica de las ciencias explicativas? Personalmente, creo que sería difícil rechazar que tenemos excelentes justificaciones epistémicas para aceptar la pertinencia formal y material de $\gamma$ (Nino, 2017, p. 190). ${ }^{35}$ Esto se debe, ante todo, a que las razones en favor de la corrección de un concepto que se obtienen mediante metodologías científicas de evaluación y sustentación, tienen preferencia epistémica como formas de averiguar cómo es el mundo

${ }^{34}$ Nótese que esta afirmación sobre las propiedades neurológicas no supone un compromiso ni con un reduccionismo ontológico ni con un monismo ontológico. Una concepción emergentista ontológica, por ejemplo, también puede dar sostén a una tesis como esta. Por lo demás, aquí no se está defendiendo una concepción ontológica, sino metodológica.

35 ¿Y si ocurriera que hay aún otras teorías explicativas que utilizan otros conceptos? Pareciera que tendríamos que responder lo mismo. Si asumiéramos esto, podríamos decir con Carlos Santiago Nino: "Es posible que un análisis [más detallado] [...] conduzca a fundamentar la conveniencia de contar incluso con varios conceptos descriptivos de sistema jurídico, para satisfacer finalidades teóricas que no pueden ser satisfechas por un único concepto" (Nino, 2017, p. 192). 
y sus partes (una de las cuales es el Derecho). ${ }^{36}$ En cambio, no comparten dicho estatus las razones o justificaciones epistémicas habilitadas por la metodología práctico-explicativa y la metodología del porqué.

En otras palabras, si las bases o razones para afirmar la corrección material de un cierto concepto clasificatorio dependen de las relaciones que este mantiene con ciertas fértiles teorías científicas propiamente explicativas y que un concepto sea materialmente correcto es condición necesaria o, incluso, es equivalente a que el concepto muestre la naturaleza de algo o lo que ese algo es, entonces la metodología de Rodríguez-Blanco para identificar el concepto de Derecho no nos brinda justificaciones epistémicas para considerar que ese concepto de Derecho muestre la naturaleza del Derecho. Es más, si un tal concepto se opusiese a otro concepto de Derecho, o a varios, que sean materialmente correctos dada sus varias relaciones con fértiles teorías propiamente explicativas, entonces deberíamos preferir estos a aquel, en lo que respecta al problema de la naturaleza del Derecho.

Obviamente, es posible que el concepto de Derecho identificado mediante la metodología propuesta por Rodríguez-Blanco también nos revele cosas interesantes del mundo social y cómo y por qué sus agentes actúan en la forma en que lo hacen: ${ }^{37}$ de seguro que un tal concepto sugiere características de la acción humana y utilizarlo como parte de una teoría explicativa no sólo es posible, sino que podría ser sumamente provechoso. ${ }^{38}$ No obstante, esto no es lo mismo que decir que mediante ese concepto se da respuesta al problema de la naturaleza del Derecho. Las razones para creer que un cierto concepto señala lo que es el Derecho, no se obtienen mediante la referida metodología. Empero, me parece que es justamente eso a lo que aspiraría Rodríguez-Blanco con su concepción metodológica.

\footnotetext{
36 Leiter (2012, parágrafo 3, capítulo I).

37 Quiero decir: en caso que pueda ser articulado o relacionado de forma fructífera con fértiles teorías genuinamente explicativas.

38 Leiter, por cierto, podría complementar este apuntamiento de la siguiente forma: "Lo que necesita [...] Finnis [...] es un argumento que explica por qué las 'necesidades' concernientes al concepto de Derecho son las vinculadas a las exigencias de la razón práctica" (Leiter, 2012 , p. 258) y no, por ejemplo, a las de potentes teorías explicativas.
} 
Naturaleza y concepto del Derecho a los ojos de Rodríguez-Blanco. Un análisis...

\section{Un epílogo}

Concluiré estos comentarios explicitando algo del programa filosófico que le subyace.

Cuenta Lakatos que en la Europa de los siglos XVII y XVIII existió un debate científico que ilustra una cuestión metodológica clave que ha influido varias metateorías filosóficas posteriores (incluyendo la que he asumido en este texto para analizar las ideas de Rodríguez-Blanco). La disputa en cuestión fue entre cartesianos y newtonianos. Es obvio que aquí no puedo reconstruir en detalle este suceso histórico, que estuvo pletórico de complejos análisis matemáticos y multitud de pruebas observacionales y experimentales. En cambio, me interesa rescatar un punto: uno de los argumentos que el cartesianismo esgrimió es que sus supuestos ontológicos podían ser "demostrados metafísicamente", ante todo porque se reputaban intuitivamente claros y distintos o se deducían de tesis - aún más abstractas - claras y distintas. Esto no podía decirse, señalaron, de los supuestos ontológicos del newtonianismo. Ante todo, estaba en la picota la existencia de la gravedad, entendida como una tendencia inmanente presente en todas las instancias del universo: era acusada de idea absurda, indemostrable y - por ende- increíble para cualquier persona "con algún raciocinio" (Lakatos, 1989, pp. 258-262).

Durante un tiempo, los newtonianos — con Newton a la cabezaintentaron demostrar su ontología. Los resultados de tales esfuerzos no fueron muy satisfactorios. Sin embargo, un argumento diferente terminó imponiéndose. Podríamos reconstruir sintéticamente ese argumento de la siguiente forma: si ciertas hipótesis $\beta$ sobre cómo se dan los procesos físicos y cosmológicos suponen necesariamente ciertas tesis ontológicas a sobre lo que hay en el mundo, y de $\beta$ se infieren implicaciones contrastables que superan de mejor manera las pruebas experimentables y observacionales frente a otras propuestas teóricas, entonces, no sólo $\beta$ sino también a son aceptables y están justificadas. La física newtoniana es mucho más exitosa que la cartesiana al explicar y describir los fenómenos físicos. Por ende, los supuestos ontológicos de la primera son preferibles a los de la segunda, no teniendo importancia si pueden ser metafísicamente demostrados o incluso si son compatibles con lo intuitivamente evidente. Es claro que lo 
que sucedió en este debate fue una variación meta-teórica en la clase de cuestiones que se consideraban epistémicamente relevantes para evaluar la corrección de una conjetura sobre qué hay en el mundo (Lakatos, 1989, pp. 258-262). Si se quiere, puede denominarse a este cambio naturalismo metodológico.

Esta dirección fue presupuesta en las páginas precedentes al analizar y criticar la propuesta metodológica de Rodríguez-Blanco: que esté justificado afirmar que un concepto establece correctamente lo que hay, supone necesariamente una conexión metodológica con relevantes y fértiles programas de investigación científica. Otros criterios (como la representación del punto de vista de una persona que posee razonabilidad práctica) serían, cuanto mucho, accesorios en esta tarea.

Es claro que en la base de tal decisión metodológica se encuentra la valoración de que la metodología de la justificación epistémica de las ciencias empíricas sólidas - como una especie sustancialmente desarrollada de la actitud racional - tiene un estatus preferente para entender el mundo, frente a otras opciones metodológicas. En favor de tal valoración me socorren los propios resultados de las ciencias.

\section{Bibliografía}

Bowler, P. J. (1992). The Fontana history of the environmental sciences. London: Fontana.

Bunge, M. (2004). La investigación cientifica (t. M. Sacristán, $3^{\text {a }}$ ed.). México DF: Siglo XXI.

De Salama, G. A. (1996). Lógica simbólica y elementos de metodología de la ciencia. Buenos Aires: Ateneo.

Diez, J. y Moulines, C. U. (1999). Fundamentos de filosofía de la ciencia (2a ed.). Barcelona: Ariel.

Elster, J. (2010). La explicación del comportamiento social, más tuercas y tornillos para las ciencias sociales (t. H. Pons). Barcelona: Gedisa.

Ferrater Mora, J. (s. f). Diccionario de filosofía. Buenos Aires: Sudamericana.

Ferrater Mora, M. y Leblanc, H. (1992). Lógica matemática. México: Fondo de Cultura Económica. 
Naturaleza y concepto del Derecho a los ojos de Rodríguez-Blanco. Un análisis...

Finnis, J. (1992). Ley Natural y Derechos Naturales (t. C. Orrego S.). Buenos Aires: Abeledo Perrot.

Jackson, F. (1994). Armchair metaphysics. En Michael, M. y O’LearyHowthorne, J. (eds), Philosophy in Mind (pp. 23-42). New York: Springer.

Leiter, B. (2012). Naturalismo y teoría del Derecho (t. G. Battista Ratti). Madrid: Marcial Pons.

Leiter, B. y Etchemendy, M. (2017). Naturalism in Legal Philosophy. En Zalta, E. N. (ed.), The Stanford Encyclopedia of Philosophy. Stanford: Stanford University. Disponible en: https://plato.stanford.edu/archives/ sum2017/entries/lawphil-naturalism/

Lakatos, I. (1989). La metodología de los programas de investigación científica (t. J. C. Zapatero). Madrid: Alianza.

Mosterín, J. (2003). Conceptos y teorías en la ciencia. Madrid: Alianza.

Mosterín, J. \& Torreti, R. (2002). Diccionario de lógica y filosofía de la ciencia. Madrid. Alianza.

Nino, C. S. (2017). La validez del derecho. Buenos Aires: Astrea.

Papineau, D. (2020). Naturalism. En Zalta, E. N. (ed.), The Stanford Encyclopedia of Philosophy. Stanford: Stanford University. Disponible en: https://plato.stanford.edu/archives/sum2020/entries/naturalism/.

Rodríguez-Blanco, V. (2021). Rastreando las críticas de Finnis al punto de vista interno de Hart: inestabilidad y el "sentido y propósito" de la acción humana en el derecho Discusiones, 26(1).

Rodríguez-Blanco, V. (2017). Entrevista. Problema, Anuario de Filosofía y Teoría del Derecho, 1(11), 547-554.

Rodríguez-Blanco, V. (2010). Objectivity in Law. Philosophy compass, 5(3), 240-249.

Rodríguez-Blanco, V. (2008). Naturalising Jurisprudence: Essays on American legal realism and naturalism in legal philosophy. Mind, 117, 1091-1094.

Rodríguez-Blanco, V. (2007). Is Finnis Wrong? Legal Theory, 13, 257-283. Rodríguez-Blanco, V. (2006). The Methodological Problem in Legal Theory: Normative and Descriptive Jurisprudence Revisited. Ratio Juris, 19(1), 26-54. 
Alejandro Guevara Arroyo

Rodríguez-Blanco, V. (2003). A defence of Hart's semantics as nonambitious conceptual analisysis. Legal Theory, 9, 99-124.

Salmon, W. (1992). Scientific Explanation. En Salmon, W. et al, Introduction to the Philosophy of Science (pp. 7-41). Indianapolis \& Cambrige: Hackett. 